\title{
An energy sensor for control of energy intake
}

\author{
BY MARK I. FRIEDMAN
}

Monell Chemical Senses Center, 3500 Market Street, Philadelphia, PA 19104, USA

It has been known for many years that laboratory rats adjust food intake in response to changes in the energy content of their diet and thereby maintain energy intake at a constant level (Adolph, 1947). Control of energy intake has been observed in other species, including human subjects, and provides an important means for maintaining energy balance, especially in species with a varied food supply (Collier, 1986). This ability to maintain energy intake in the face of changes in dietary energy content implies the existence of a feedback signal that communicates changes in energy intake to mechanisms that control eating behaviour.

Compensation for changes in the energy density of food appears to depend on the ability to catabolise the source of dietary energy (see Friedman, 1991). This suggests that the feedback signal controlling energy intake is not in the food, but rather is generated in the oxidation of metabolic fuels when the energy from food is finally realized (Friedman, 1991). Energy expenditure and the status of body energy stores also determine the number of kilojoules consumed. It is possible that these metabolic determinants influence energy intake via a signal derived from the breakdown of energy-yielding substrate (Friedman, 1991, 1995). In other words, control of energy intake, whether in response to changes in dietary energy content or in the service of body energy balance, is based on the ability to sense energy.

In the present paper, I review evidence for a mechanism for the control of eating behaviour that is based on the detection of energy in its most fundamental biochemical form. The nature of the metabolic signal, the location of its sensor, and the means by which the signal is communicated to the brain are outlined. How such a mechanism might operate as an energy sensor to control energy intake in response to changes in dietary energy density, energy expenditure, and energy storage is then discussed.

\section{METABOLIC CONTROL OF FOOD INTAKE}

What is the nature of the metabolic signal that controls food intake? The best-known theories focus on signals associated with glucose and fat metabolism (see Friedman \& Stricker, 1976). According to 'glucostatic' and 'lipostatic' theories, intracellular glucose utilization or blood glucose level (Mayer, 1955; Campfield \& Smith, 1990) provide one type of stimulus for eating behaviour, whereas body fat or fatty acid oxidation (Le Magnen et al. 1973; Harris \& Martin, 1984) provide another. A different line of thought about the metabolic signal for food intake, which has evolved in parallel with the traditional glucostatic and lipostatic theories, vests control at a level of metabolism downstream from pathways involved in processing specific substrates. In this case, the signal is generated in the final oxidative pathways of metabolism where individual substrates are indistinguishable and where processes for the production of biochemically-useful energy predominate.

Ugolev and his colleagues (Kassil et al. 1970) were the first to suggest that the control of food intake is based on metabolic events in the final pathways of energy metabolism. As 
they put it, '... regulation of food consumption should be attributed, above all, to the liberation of basic energy ...' Others have emphasized a control of food intake based on a signal associated with available energy rather than with the status of individual substrates (Panksepp, 1971; Booth, 1972), although the nature of the stimulus was not specified. Ugolev (Kassil et al. 1970) emphasized tricarboxylic-acid-cycle intermediates as signals for control of food intake. More recently, hypotheses concerning the source of the signal have centred on fuel oxidation and the formation of ATP through the process of oxidative phosphorylation (Nicolaidis, 1974; Friedman \& Stricker, 1976; Friedman et al. 1986; Nicolaidis \& Even, 1990; Langhans \& Scharrer, 1992). Findings which will be described favour the latter hypothesis.

In order for a metabolic event to influence energy intake, there must be a sensory system that detects the metabolic signal and transmits information about it to the brain systems that govern eating behaviour (Friedman, 1990). Evidence indicates that there are metabolic sensors in the brain and in the liver that could serve this function. Metabolic sensors in the brain appear to mediate eating responses under emergency conditions (Epstein et al. 1975) because they seem to be activated only under extreme deficits in energy supply. In addition, the brain is well-protected from fluctuations in the energy supply under normal circumstances in which control of energy intake is observed. In this regard, an hepatic metabolic sensor may be better suited for control of food intake under normal conditions because liver metabolism fluctuates dramatically with normal feeding and fasting cycles. Empirical data appear to agree with this conclusion.

Russek $(1963,1981)$ was the first to suggest that the liver detected metabolic signals that control eating behaviour. Research on the liver's role in the control of food intake has focused primarily on the inhibition of eating or satiety, probably because the organ is in an obviously good position to assess the consequences of ingestion and provide a negative feedback signal (Novin \& VanderWeele, 1977). The most direct demonstration of the liver's role in satiety comes from studies showing that infusion of physiological amounts of glucose into the hepatic portal vein reduces food intake of freely-feeding rats more effectively than does infusion into the jugular vein (Tordoff \& Friedman, 1986; Tordoff et al. 1989). Other substrates and agents that suppress food intake (e.g. fructose, glucagon, amphetamine and hypertonic saline $(>9 \mathrm{~g} \mathrm{NaCl} / \mathrm{l}))$ are also more effective when given by the hepatic portal rather than the jugular route (e.g. Tordoff et al. 1982, 1986; Tordoff \& Friedman, 1988; Geary et al. 1993).

Less work has been done on the role of the liver in initiating eating behaviour. The possibility that hepatic signals trigger feeding was first examined using 2-deoxy-D-glucose (2DG), a glucose analogue that stimulates food intake; however, experiments to localize the behavioural effect of 2DG to liver produced equivocal results (Novin et al. 1973; Russell \& Mogenson, 1976), most probably because 2DG is not confined to the liver and has direct effects in the brain.

We have used a fructose analogue, 2,5-anhydro-D-mannitol (2,5-AM), to study the hepatic mechanism for initiation of eating behaviour (Tordoff et al. 1988, 1991). Because fructose does not cross the blood-brain barrier, 2,5-AM was not expected to affect cerebral metabolism as do glucose analogues. 2,5-AM is structurally identical to the furanose form of fructose except for the lack of the C-2 hydroxyl group (making it a 2-deoxy-D-fructose). Experiments on the mechanisms of action of 2,5-AM have provided considerable support that a hepatic signal associated with changes in oxidative phosphorylation initiates eating behaviour. This work raises the possibility that an energy sensor is involved in the control of energy intake. 


\section{A HEPATIC ENERGY SIGNAL FOR CONTROL OF EATING BEHAVIOUR}

Administration of 2,5-AM stimulates eating in rats during the daylight hours when these nocturnal animals normally eat very little (Tordoff et al. 1988, 1991). The eating response to 2,5-AM usually occurs with $90 \mathrm{~min}$ after administration. Although the effect is seen as an increase in intake $(\mathrm{g})$, this increase reflects a greater proportion of animals initiating eating behaviour. Thus, 2,5-AM treatment causes rats to eat.

\section{An hepatic sensor}

A number of lines of evidence strongly indicate that 2,5-AM stimulates eating by its action in the liver. Direct and convincing evidence for an hepatic site of action for the effect of 2,5-AM on eating behaviour comes from experiments which compare hepatic portal vein and jugular vein infusions of the analogue. Portal infusion of 2,5-AM stimulates eating behaviour more rapidly and at lower doses than does jugular infusion of the analogue (Tordoff et al. 1991). Indeed, at one dose tested, portal administration elicited an eating response whereas jugular infusion was ineffective. Because portal vein infusion is made through a catheter placed close to the liver, this is the first organ exposed to 2,5-AM and consequently the organ that is exposed to the highest concentration of the analogue. In contrast, the liver is not preferentially exposed to 2,5-AM infused into the jugular vein.

There is no indication that the analogue has direct action in the brain, the only other site where metabolic inhibitors (i.e. glucose analogues) have been shown to act to elicit eating behaviour. Consistent with the failure of fructose to cross the blood-brain barrier, the fructose analogue also does not appear to be taken up into cerebral tissue. No 2,5-AM is found in brain after rats are given $\left[{ }^{14} \mathrm{C}\right] 2,5-\mathrm{AM}$ (Tordoff $e$ t al. 1991). Similarly little if any $\left[1-{ }^{3} \mathrm{H}\right] 2,5-\mathrm{AM}$ was found in the brains of rats that had ingested the analogue in their drinking water for $24 \mathrm{~h}$ (Dills et al. 1992). Moreover, brain tissue apparently lacks fructokinase (EC 2.7.1.4) which is necessary for metabolism of 2,5-AM (see Dills et al. 1992). Thus, it is not surprising that injection of 2,5-AM does not produce any signs of behavioural impairment (ataxia) or unusual metabolic perturbations (e.g. hyperglycaemia), which are indicative of a cerebral energetic emergency. In light of the lack of brain uptake of 2,5-AM, the finding that portal vein infusion of 2,5-AM is more effective than jugular vein infusion constitutes prima facie evidence that the analogue acts in the liver to stimulate eating behaviour.

Uptake and metabolism data provide additional evidence for an hepatic site of action for the effect of 2,5-AM on eating behaviour. Relative to other tissues, the liver is the primary site for uptake of the analogue and the time-course of uptake in liver best matches that of the eating response (Tordoff et al. 1991). The amount of 2,5-AM that accumulates in the liver after an oral dose that stimulates eating is similar to the amount infused in the portal vein when the eating response is initiated (Tordoff et al. 1991). The eating response to 2,5-AM is tied to its phosphorylation in liver (see p. 44). Accordingly, the proportion of 2,5-AM phosphorylated relative to the amount taken up is highest in liver compared with other tissues (Dills et al. 1992), and the time-course of phosphorylation in liver parallels the eating response (Tordoff et al. 1991). Finally, as discussed in detail later (see pp. 44 46) there are a number of situations in which changes in liver metabolism are tied in a predictable fashion to the eating response after 2,5-AM treatment.

Results of experiments showing that hepatic branch vagotomy abolishes the eating response to low doses of 2,5-AM (Tordoff et al. 1991) provide additional support that the analogue acts in liver to elicit food intake. However, this result alone is not strong evidence because the hepatic branch contains both afferent and efferent fibres and innervates other 
tissues besides the liver. Nevertheless, the observation is consistent with an hepatic site of action.

Taken together, these results provide considerable evidence that 2,5-AM stimulates eating behaviour by acting in the liver. Identification of the liver as the site of action has enabled a more detailed investigation of the mechanism of action of 2,5-AM, starting with an examination of the metabolic effects of the analogue that might underlie the eating response.

\section{An energy stimulus}

There has been much speculation about the nature of the hepatic metabolic stimulus (or stimuli) for control of food intake. Russek (1981) proposed various metabolic variables associated with hepatic metabolism (e.g. glycogen level, pyruvate concentration), but convincing evidence for any of these is lacking. Glucagon has been implicated in the hepatic control of feeding (Geary et al. 1993), but, although it is thought to act via changes in hepatic fuel metabolism, the metabolic signal has not been specified. Other hypotheses about the hepatic metabolic stimulus have focused on an oxidative stimulus of the type described previously (Friedman \& Stricker, 1976; Langhans \& Scharrer, 1992), and studies of the mechanism of action of 2,5-AM on eating behaviour have supported a role for such a stimulus.

2,5-AM has a variety of effects on fuel metabolism. Injection of 2,5-AM alters the plasma fuel profile to one indicative of a mild fast (i.e. slight decline in glucose and increase in fatty acids, glycerol and ketone bodies; Tordoff et al. 1988), and shifts wholebody fuel metabolism from carbohydrate to fat oxidation (Park et al. 1995). Although the shift to fatty acid oxidation may restrain the eating response to 2,5-AM (Rawson et al. 1996), this and other effects at the substrate level do not appear to account for the eating response to the analogue because dietary, metabolic, pharmacological and neurological treatments that alter the behavioural response to 2,5-AM do so without affecting the metabolic response at the substrate level in any consistent pattern (Tordoff et al. 1988, 1991; Rawson et al. 1996).

Like fructose, 2,5-AM is rapidly phosphorylated in hepatocytes at the C-1 and C-6 positions to the mono- and then bisphosphate forms. However, unlike fructose, the analogue is not further metabolized through glycolysis (see Riquelme et al. 1985) and therefore, inorganic phosphate (Pi) is trapped in the phosphorylated forms of 2,5-AM. The depletion of Pi apparently compromises ATP synthesis and enhances the degradation of adenosine nucleotides through disinhibition of deaminase activity (see Rawson et al. 1994a). Consequently, liver ATP content declines after 2,5-AM treatment (Rawson et al. $1994 a$ ).

Phosphate depletion appears to be crucial for the effect of 2,5-AM on liver ATP and eating behaviour because administration of exogenous phosphate prevents both the decrease in hepatic ATP and the increase in feeding produced by 2,5-AM injection (Rawson \& Friedman, 1994). These effects of phosphate loading are specific to the phosphate anion because administration of equiosmotic $\mathrm{NaCl}$ did not affect either the eating response or the decrease in hepatic ATP after 2,5-AM. Phosphate loading did not interfere with the eating response to other treatments indicating that the lack of response to 2,5-AM was not due to malaise or elicitation of competing behaviours. These findings indicate that depletion of $\mathrm{Pi}$ due to phosphate trapping is a critical intracellular factor in the 
mechanism by which 2,5-AM triggers food intake, and suggest that a decrease in liver ATP or some associated event generates a stimulus that initiates eating behaviour.

Although phosphate trapping and depletion of $\mathrm{Pi}$ are important for the mechanism of action of 2,5-AM, the decline in ATP rather than in Pi appears to be involved in triggering the eating response. L-Ethionine (Eth), an analogue of the amino acid methionine, also decreases ATP levels in liver (Smith et al. 1987), but by trapping the adenine moiety of ATP (Shull et al. 1966) instead of by trapping phosphate (indeed, Pi increases after Eth treatment). Administration of Eth also stimulates feeding behaviour in rats at doses that decrease hepatic ATP (Rawson et al. 1994b). Comparison of the effect of Eth on food intake with that on hepatic ATP measured by in vivo ${ }^{31} \mathrm{P}$ magnetic resonance spectroscopy in anaesthetized rats given the same dose (Smith et al. 1987) suggests that the change in behaviour follows a time-course similar to that in hepatic ATP. Because Eth depletes ATP in a different way to that of $2,5-\mathrm{AM}$, these results provide independent evidence that decreased ATP triggers feeding, and lend considerable support to the hypothesis that changes in ATP provide a signal controlling feeding behaviour.

The decline in liver ATP in rats given 2,5-AM by intraperitoneal injection is nearly maximal within $15 \mathrm{~min}$ and persists for at least $90 \mathrm{~min}$, whereas the eating response under identical conditions occurs primarily between 15 and 45 min after treatment (Koch et al. $1995 b$ ). Thus, the decrease in liver ATP precedes the eating response as would be expected if this metabolic event provided a stimulus for the behaviour. If access to food is delayed for $4 \mathrm{~h}$ after injection of 2,5-AM, liver ATP returns to baseline levels and the eating response on refeeding is attenuated compared with that observed when access is delayed for $60 \mathrm{~min}$ at which time ATP is still decreased (J. E. Koch \& M. I. Friedman, unpublished results). This finding suggests that a decease in liver ATP is a necessary condition for the timing of the eating response to 2,5-AM. The amount of food consumed after 2,5-AM treatment, however, may be determined by other factors because food consumption does not immediately restore liver ATP (J. E. Koch \& M. I. Friedman, unpublished results). This finding is consistent with previously described behavioural data suggesting that 2,5-AM affects meal initiation.

The increase in fat fuel oxidation after 2,5-AM injection appears to restrain the eating response to the analogue because feeding a high-fat low-carbohydrate diet, which enhances fatty acid oxidation, prevents the increase in eating behaviour, whereas administration of a fatty acid oxidation inhibitor potentiates it (Rawson et al. 1996). Rats fed on the high-fat low-carbohydrate diet show a smaller reduction in liver ATP after 2,5-AM than do rats fed on a low-fat high-carbohydrate diet (Koch et al. 1995a), suggesting that increased fatty acid oxidation attenuates the eating response to 2,5-AM because it also ameliorates the decline in liver ATP produced by the analogue.

To provide a picture of the relationship between liver ATP and eating behaviour after 2,5-AM treatment, we performed regression analyses of data from three experiments in which both eating behaviour and liver ATP were assessed in the same animals. In each experiment, the eating response was measured first. After 1 week rats were given the same treatment (i.e. saline $(9 \mathrm{~g} \mathrm{NaCl} / \mathrm{l})$ or 2,5-AM) as used in the eating test, and $30 \mathrm{~min}$ after that livers were freeze-clamped for analysis of ATP. These experiments examined the time-course and the effects of diet composition on both the behavioural and metabolic effects of 2,5-AM in over 100 animals in twelve treatment groups that were maintained on one of three different diets and were given either saline or one of two doses of 2,5-AM.

Because 2,5-AM administration triggers eating behaviour, we examined the relationship between average liver ATP concentration of the twelve treatment groups and the likelihood of meal initiation, as reflected in the proportion of rats in that group that ate at 


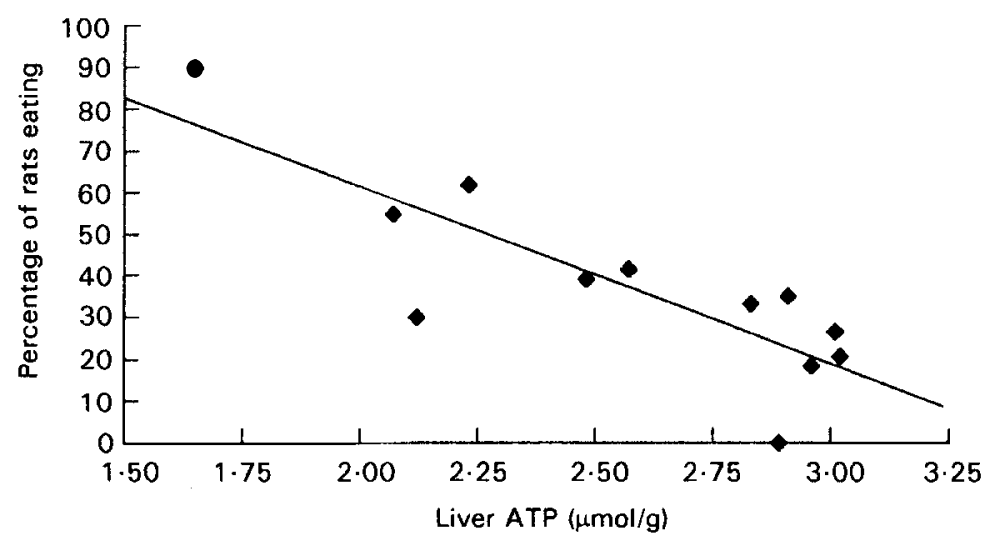

Fig. 1. Relationship between the probability of initiating eating behaviour and liver ATP concentration in rats. Each point represents data from a different group of eight to ten rats fed one of three diets and injected with one of two doses of 2,5-anhydro-D-mannitol (2,5-AM) or saline $(9 \mathrm{~g} \mathrm{NaCl})$. Rats were tested first for the eating response to injection of 2,5-AM, and 1 week later liver ATP was measured $30 \mathrm{~min}$ after the same treatment but in the absence of food. The regression analysis was performed using the mean ATP value for each group and the percentage of rats in each group eating at least $0.5 \mathrm{~g}$ within the first hour after injection. Percentage values were subjected to an arcsine correction. $r$ 0.83 .

least $0.5 \mathrm{~g}$ within $1 \mathrm{~h}$ after injection. This analysis (Fig. 1) showed a striking inverse correlation between average liver ATP and the percentage of rats eating; approximately $70 \%$ of the variance in the likelihood of eating can be accounted for by liver ATP level. Similar correlations were found when eating behaviour was considered over a longer interval $(2 \mathrm{~h})$ and when 0.3 or $1.0 \mathrm{~g}$ were used as criteria for meal initiation. Average liver ATP and food intake showed a similar inverse correlation. The results of these analyses strengthen the conclusion that initiation of eating after 2,5-AM treatment is associated with a decrease in liver ATP.

Whether changes in liver ATP can account for variations in eating behaviour under other conditions remains to be seen. Rats with experimental diabetes mellitus, which are extremely hyperphagic, are reported to have a relatively low liver ATP content (Kang, 1979). Fasting, which clearly stimulates eating behaviour, also reduces liver ATP of rats (Start \& Newsholme, 1968; Cunningham et al. 1986). This effect is seen within $12 \mathrm{~h}$ of food deprivation during the day-time (Start \& Newsholme, 1968) when rats normally eat very little, suggesting that normally-occurring changes in liver ATP might provide a signal to eat under ad libitum feeding conditions.

\section{Transmission of the hepatic metabolic stimulus}

Very little information is available concerning the mechanisms by which metabolic events in liver are transduced into a signal for the nervous system (Langhans \& Scharrer, 1992; Friedman \& Tordoff, 1996). Once transduced, however, it appears that vagal afferents from the liver to the brain carry the metabolic signal that triggers eating behaviour after injection of 2,5-AM. Hepatic portal vein infusion of 2,5-AM increases the firing rate of afferent fibres in the hepatic branch of the vagus (A. Niijima, personal communication; E. Scharrer, personal communication), and hepatic vagotomy prevents the eating response to low doses of 2,5-AM (Tordoff et al. 1991). Lesions of the parabrachial nucleus, which is a relay for the central vagal afferent system, prevent the eating response to 2,5-AM (Grill et al. 1995). 
Injection of 2,5-AM increases neuronal activity in central vagal afferent pathways (as measured by fos-immunocytochemistry). As for the eating response, this activation is prevented by vagotomy (Ritter et al. 1994) and by feeding a high-fat diet (Horn \& Friedman, 1996), and is potentiated by concomitant administration of a fatty acid oxidation inhibitor (Horn \& Friedman, 1995). Perivagal application of capsaicin, which destroys certain small-diameter unmyelinated sensory fibres, also prevents 2,5-AM-induced eating and reduces neuronal activation in selected central vagal afferent projection areas after 2,5AM treatment (C. C. Horn \& M. I. Friedman, unpublished results). These results strongly suggest that a subpopulation of small vagal afferent neurons carries the hepatic signal to initiate eating behaviour.

\section{IMPLICATIONS FOR THE CONTROL OF ENERGY INTAKE}

The studies described previously suggest the existence of a sensor that detects changes in cellular energy production and relays this information to the brain to control eating behaviour in rats. This mechanism could serve as an energy sensor for control of energy intake. Because the metabolic events to which it responds are at a level of metabolism where individual macronutrients are indistinguishable, this sensor would be sensitive to the production of energy from different metabolic fuels. Thus, it would monitor energy, not specific substrates. Because the sensor is in the liver, which is central in the processing of metabolic fuels, it would be responsive to changes in the supply of energy-yielding substrates from either the gastrointestinal tract or internal energy stores. It could, therefore, play a role in the control of energy intake in response to changes in dietary energy density or in energy expenditures or storage.

Although there are no data directly linking an hepatic energy sensor to the control of energy intake, such a mechanism could help explain the appropriate adjustments in energy intake in response to environmental and metabolic demands as well as the apparent failures in control of energy intake under certain dietary or metabolic conditions (for more detailed discussion, see Friedman, 1991, 1995, 1997). Because energy production in the sensor must depend on its fuel supply, the response to changes in the energy density of food might simply reflect a decrease or increase in the delivery of energy-yielding substrate to the sensor. However, metabolic fuels can be utilized by tissues which do not have a sensor and can be utilized in the sensory organ or other tissues for storage instead of energy production. Therefore, the energy signal within the sensor may vary depending on how fuels are partitioned among tissues and metabolic pathways. Thus, thermogenesis and exercise increase energy intake because fuels are redirected toward heat production and muscular activity and away from the sensor.

Seemingly inappropriate changes in energy intake can also be understood in terms of an energy sensor that operates within the context of a metabolic regulatory system characterized by fuel partitioning. Overconsumption of energy during the development of obesity, for example, can be seen as a response to a primary shift in fuel partitioning toward fat storage and away from oxidative pathways. Dietary factors which promote hyperphagia may operate in the same manner; for instance, overeating which results from incomplete compensation for an increase in dietary energy density may be due to such a 'loss' of ingested fuels into storage. It is important to note that, according to the perspective outlined here, the hyperphagia associated with obesity is due not to a failure of the energy sensor, but to disturbances in the partitioning of metabolic fuels. Overeating does not result because the energy sensor is ineffective, but because it works too well: it sends the 
appropriate signal to eat in response to a decrease in its production of energy, whether the decrement is due to a paucity of fuels from an energetically-dilute diet, an increased expenditure for thermogenesis or exercise, or the deposition of fuels into adipose tissue.

\section{SUMMARY AND CONCLUSIONS}

Control of energy intake, either in response to changes in the energy content of food or in energy expenditures and storage, is based on the detection of a feedback signal generated in the processing of metabolic fuels for energy. Evidence from studies of the fructose analogue, 2,5-AM, indicates a sensor in liver responds to changes in intracellular ATP or some closely associated event and communicates this information to the brain via vagal afferent neurons. Such a mechanism could serve as the energy sensor which controls energy intake.

\section{REFERENCES}

Adolph, E. F. (1947). Urges to eat and drink in rats. American Journal of Physiology 151, 110-125.

Booth, D. A. (1972). Postabsorptively induced suppression of appetite and the energostatic control of feeding. Physiology and Behavior 9, 199-202.

Campfield, L. A. \& Smith, F. J. (1990). Systemic factors in the control of food intake. Evidence for patterns as signals. In Handbook of Behavioural Neurobiology: Food and Water Intake, pp. 183-206 [E. M. Stricker, editor]. New York: Plenum Publishing.

Collier, G. (1986). The dialogue between the house economist and the resident physiologist. Nutrition and Behavior 3, 9-26.

Cunningham, C. C., Malloy, C. R. \& Radda, G. K. (1986). Effects of fasting and acute ethanol administration on the energy state of in vivo liver as measured by ${ }^{31}$ P-NMR spectroscopy. Biochimica et Biophysica Acta 885 , $12-22$.

Dills, W. L., Murphy-Kothe, J. \& Klinger, J. (1992). Absorption, excretion and tissue distribution of 1-[ $\left.{ }^{3} \mathrm{H}\right]-2,5-$ anhydro-D-mannitol in female Wistar rats. Biochemical Archives 8, 69-74.

Epstein, A. N., Nicolaidis, S. \& Miselis, R. (1975). The glucoprivic control of food intake and the glucostatic theory of feeding behavior. In Neural Integration of Physiological Mechanisms and Behavior, pp. 148-168 [G. J. Mogenson and F. R. Calaresu, editors]. Toronto: University of Toronto Press.

Friedman, M. I. (1990). Making sense out of calories. In Handbook of Behavioral Neurobiology: Food and Water Intake, pp. 513-529 [E.M. Stricker, editor]. New York: Plenum Publishing.

Friedman, M. I. (1991). Metabolic control of calorie intake. In Chemical Senses. vol. 4, Appetite and Nutrition, pp. 19-38 [M. I. Friedman, M. G. Tordoff and M. R. Kare, editors]. New York: Marcel Dekker.

Friedman, M. I. (1995). Control of energy intake by energy metabolism. American Journal of Clinical Nutrition 62, Suppl., 1096S-1100S.

Friedman, M. I. (1997). Fuel partitioning and food intake. American Journal of Clinical Nutrition (In the Press)

Friedman, M. L. \& Stricker, E. M. (1976). The physiological psychology of hunger: A physiological perspective. Psychological Review 83, 409-431.

Friedman, M. I. \& Tordoff, M. G. (1996). Hepatic metabolic signal for control of food intake: Stimulus generation, signal transduction and neural transmission. In Liver Innervation, pp. 373-380 [T. Shimazu, editor]. London: John Libby.

Friedman, M. I., Tordoff, M. G. \& Ramirez, I. (1986). Integrated metabolic control of food intake. Brain Research Bulletin 17, 855-859.

Geary, N., Le Sauter, J. \& Moh, U. (1993). Glucagon acts in liver to control spontaneous meal size in rats. American Journal of Physiology 264, R116-R122.

Grill, H. J., Friedman, M. I., Norgren, R., Scalera, G. \& Seeley, R. (1995). Parabrachial nucleus lesions impair the feeding response elicited by 2,5-anhydro-D-mannitol. American Journal of Physiology 268, R676-R682.

Harris, R. B. S. \& Martin, R. J. (1984). Lipostatic theory of energy balance: Concepts and signals. Nutrition and Behavior 1, 253-275.

Horn, C. C. \& Friedman, M. I. (1995). Synergistic increase in food intake and brain Fos-like immunoreactivity (Fos-li) after treatment with methyl palmoxirate (MP) and 2,5-anhydro-D-mannitol. Obesity Research 3, Suppl. 3, 330S. 
Horn, C. C. \& Friedman, M. I. (1996). Diet-related increases in food intake and brain Fos-like immunoreactivity (Fos-li) after treatment with 2,5-anhydro-D-mannitol (2,5-AM). FASEB Journal 10, A823.

Kang, S. S. (1979). The effects of dietary sucrose and streptozotocin-diabetes on blood and liver constituents. Nutrition and Metabolism 23, 327-334.

Kassil, V. G., Ugolev, A. M. \& Chernigovskii, V. N. (1970). Regulation of selection and consumption of food and metabolism. Progress in Physiological Sciences 1, 387-404.

Koch, J. E., Graczyk-Millbrandt, G., Osbakken, M. D., Blum, H., Ketchum, M. A., Nuss, J. L. \& Friedman, M. I. (1995a). Diet-related effects of 2,5-anhydro-D-mannitol on food intake and liver ATP. Obesity Research 3, Suppl. 3, 375S.

Koch, J. E., Ulrich, P., Nuss, J. \& Friedman, M. I. (1995b). Time course of the effect of 2,5-anhydro-D-mannitol on food intake and liver ATP. Obesity Research 3, Suppl. 3, 375S.

Langhans, W. \& Scharrer, E. (1992). Metabolic control of eating. World Review of Nutrition and Dietetics 70, $1-67$.

Le Magnen, J., Devos, M., Gaudilliere, J. P., Louis-Sylvestre, J. \& Tallon, S. (1973). Role of a lipostatic mechanism in regulation by feeding of energy balance in rats. Journal of Comparative and Physiological Psychology 84, 1-23.

Mayer, J. (1955). Regulation of energy intake and the body weight: the glucostatic theory and lipostatic hypothesis. Annals of the New York Academy of Sciences 63, 15-42.

Nicolaidis, S. (1974). Short-term and long-term regulation of energy balance. Proceedings of the 26th International Congress of Physiological Sciences (New Delhi) 10, 122-123.

Nicolaidis, S. \& Even, P.C. (1990). The ischymetric control of feeding. International Journal of Obesity 14, Suppl. 3, 35-52.

Novin, D. \& VanderWeele, D. A. (1977). Visceral involvement in feeding: there is more to regulation than the hypothalamus. Progress in Psychobiology and Physiological Psychology 7, 193-241.

Novin, D., VanderWeele, D. A. \& Rezek, M. (1973). Infusions of 2-deoxy-D-glucose into the hepatic portal system causes eating: evidence for peripheral glucoreceptors. Science 181, 858-860.

Panksepp, J. (1971). Effects of fats, proteins, and carbohydrates on food intake in rats. Psychonomic Monograph Supplement 4, 86-95.

Park, C. R., Seeley, R. J., Benthem, L., Friedman, M. I. \& Woods, S. C. (1995). Whole body energy expenditure and fuel oxidation after 2,5-anhydro-D-mannitol administration. American Journal of Physiology 268, R299R302.

Rawson, N. E., Blum, H., Osbakken, M.D. \& Friedman, M. I. (1994a). Hepatic phosphate trapping, decreased ATP and increased feeding after 2,5-anhydro-D-mannitol. American Journal of Physiology 266, R112-R117.

Rawson, N. E. \& Friedman, M. I. (1994). Phosphate-loading prevents the decrease in ATP and increase in food intake produced by 2,5-anhydro-D-mannitol. American Journal of Physiology 266, R1792-R1796.

Rawson, N. E., Ulrich, P.M. \& Friedman, M. I. (1994b). L-Ethionine, an amino acid analogue, stimulates eating in rats. American Joumal of Physiology 267, R612-R615.

Rawson, N. E., Ulrich, P.M. \& Friedman, M.I. (1996). Fatty acid oxidation modulates the eating response to the fructose analogue, 2,5-anhydro-D-mannitol. American Journal of Physiology 271, R144-148.

Riquelme, P. T., Kneer, N. M., Wernette-Hammond, M. E. \& Lardy, H. S. (1985). Inhibition of glycolysis in isolated rat hepatocytes and in Ehrlich ascites cells. Proceedings of the National Academy of Sciences USA 82, $78-82$.

Ritter, S., Dihn, T. T. \& Friedman, M. I. (1994). Induction of Fos-like immunoreactivity (Fos-li) and stimulation of feeding by 2,5-anhydro-D-mannitol (2,5-AM) require the vagus nerve. Brain Research 646, 53-64.

Russek, M. (1963). An hypothesis on the participation of hepatic glucoreceptors in the control of food intake. Nature 197, 79-80.

Russek, M. (1981). Current status of the hepatostatic theory of food intake control. Appetite 2, 137-143.

Russell, P. J. D. \& Mogenson, G. J. (1976). Drinking and feeding induced by jugular and portal infusions of 2deoxy-D-glucose. American Joumal of Physiology 229, 1014-1018.

Shull, K. H., McConomy, J., Vogt, M., Castillo, A. \& Farber, E. (1966). Journal of Biological Chemistry 241, 5060-5070.

Smith, L. J., Murphy, E., Gabel, S. A. \& London, R. E. (1987). In vivo ${ }^{31}$ P NMR studies on the hepatic response to L-ethionine in anesthetized rats. Toxicology and Applied Pharmacology 88, 346-353.

Start, C. \& Newsholme, E. A. (1968). The effects of starvation and alloxan-diabetes on the contents of citrate and other metabolic intermediates in rat liver. Biochemical Journal 107, 411-415.

Tordoff, M. G. \& Friedman, M. I. (1986). Hepatic portal glucose infusions decrease food intake and increase food preference. American Journal of Physiology 251, R191-R196.

Tordoff, M. G. \& Friedman, M. I. (1988). Hepatic control of feeding: effect of glucose, fructose and mannitol infusion. American Journal of Physiology 254, R969-R976.

Tordoff, M. G., Hopfenbeck, J., Butcher, L. L. \& Novin, D. (1982). A peripheral locus for amphetamine anorexia. Nature 279, 148-150.

Tordoff, M. G., Rafka, R., DiNovi, M. J. \& Friedman, M. I. (1988). 2,-5-Anhydro-D-mannitol: A fructose analogue that increases food intake in rats. American Journal of Physiology 254, R150-R153. 
Tordoff, M. G., Rawson, N. \& Friedman, M. I. (1991). 2,5-Anhydro-D-mannitol acts in liver to initiate feeding. American Journal of Physiology 261, R283-R288.

Tordoff, M. G., Schulkin, J. \& Friedman, M. I. (1986). Hepatic contribution to satiation of salt appetite in rats. American Journal of Physiology 251, R1095-R1102.

Tordoff, M. G., Tluczek, J. P. \& Friedman, M. I. (1989). Effect of hepatic portal glucose concentration on food intake and metabolism. American Joumal of Physiology 257, R1474-R1480. 